\title{
L'utilisation des tests génétiques par les assureurs
}

\section{Marie-Angèle Hermitte}

M.A. Hermitte : Cnrs-CRDP, 12, place du Panthéon, 75005 Paris, France.

L'utilisation des tests génétiques débouchant sur une médecine prédictive poussée à son paroxysme a suscité, dès l'origine, de nombreuses inquiétudes. Le test génétique $n^{\prime}$ est-il, entre les mains de l'assureur, qu'un outil de plus ou une machine de nature plus dangereuse? Qu'adviendra-t-il lors de la remise en chantier des lois sur la bioéthique? La crainte des scientifiques se cristallise autour du refus de réalisation de ces tests par la population.

Pourtant, ce sont les campagnes de dépistage qui devraient être au cœur du débat.
Ce texte se fonde sur une étude financée par la MIRE et réalisée dans le cadre du département Science et droit du Centre de recherches en droit privé, avec D. Dibie et C. Noiville.

\section{TIRÉS À PART}

M.A. Hermitte.

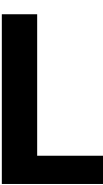
e projet génome humain fut d'abord un rêve scientifique avec ses espoirs de connaissances et de guérisons. II aboutit mécaniquement à la connaissance de gènes impliqués dans des maladies et à la fabrication de tests de diagnostic, disponibles sur le marché bien avant que la thérapeutique ne soit au rendez-vous si elle y est un jour. Ces tests sont utilisés par leurs concepteurs pour vérifier, dans des familles atteintes par une maladie héréditaire, la présence du gène chez tel ou tel individu (médecine individuelle), et pour constituer de vastes populations de familles concernées acceptant d'entrer dans des protocoles de recherche. On s'est inquiété dès l'origine de ces pratiques, redoutant qu'elles ne débouchent sur une analyse systématique du génome de chaque nourrisson, doté d'une «carte d'identité génétique», censé manger, fumer, se soigner comme le lui commande son "capital santé ». La médecine prédictive poussée à son terme articulerait les stratégies de santé publique et la responsabilité individuelle pour une gestion rationnelle de la santé, dans un monde transparent. Aujourd'hui que les tests de diagnostic arrivent sur le marché, on passe de la science à la société, et les nouveaux outils échappent à leurs créateurs. Le type de "prédiction" que permet la connaissance du génome d'un individu intéresse la médecine prédictive, mais aussi les employeurs qui préféreront ne pas mettre un salarié sensible à un produit chimique à un poste de travail où il est en contact avec ce produit, et les assureurs de la branche "vie", dont le métier est de sélectionner les risques des souscripteurs, en particulier leur profil de santé.

Faut-il interdire tout usage des tests génétiques en dehors de la sphère médicale en raison des risques de discrimination qu'impliquerait, par hypothèse, le fait de se fonder sur une prédiction de santé pour refuser un poste de travail ou faire payer une surprime au souscripteur d'une assurance-vie ? C'est la solution radicale que certains préconisent - et I'on constatera à cet égard un consensus curieux entre défenseurs des droits de l'homme un peu pressés de conclure et généticiens inquiets de l'image négative que leurs tests pourraient acquérir s'ils s'égaraient dans des utilisations non médicales*. Un outil technique peut-il être ainsi isolé dans la sphère médicale, retranché de la société, et à quel prix pour les autres secteurs de la société ? Quelles seraient les conséquences d'une telle décision pour l'économie du contrat d'assurance?

Car c'est en ces termes que doit être posée la question. Si les contrats d'assurance de la branche vie sont considérés comme des outils sociaux légitimes à côté de la sécurité sociale dont ils sont bien distincts, quelle est leur fonction dans la société? Le contrat d'assurance est-il un simple confort ou fait-il partie des biens premiers auxquels chacun doit avoir accès ? Quel est l'objet de ces contrats (assurer un risque qu'il faut * Résolution du Parlement européen du
16 mars 1989. Avis no 46 du CCNE. 
sélectionner pour pouvoir le tarifer)? Il faudra surtout se poser la question de savoir pourquoi le contrat d'assurance a été qualifié de contrat de bonne foi, impliquant par nature que les deux parties disposent du même niveau d'information sur le risque. Entre les mains de l'assureur, le test génétique n'est-il qu'un outil de plus venant s'insérer entre le questionnaire de santé, les tests biologiques classiques et les radios, est-il d'une nature plus dangereuse, ce qui impliquerait un régime juridique distinct ? Cet article essaie de répondre à ces questions en les examinant dans leur contexte. II s'agit de rappeler en premier lieu que l'assurance privée est distincte de la sécurité sociale qui exclut toute forme de sélection des risques, et que dans son secteur traditionnel, le droit de l'assurance, avec son principe de bonne foi paraît non seulement légitime mais indispensable. Pourtant, la rigidité de la frontière entre les deux est fragilisée par les difficultés économiques de la sécurité sociale d'une part, la volonté de l'assurance privée de s'emparer de ce marché d'autre part. Or, plus l'assurance privée empiète sur le terrain du droit à la santé, moins ses règles de fonctionnement actuelles paraissent légitimes. L'étude examine ensuite différentes propositions faites par le Conseil de l'Europe, le droit communautaire et différents droits étrangers pour aborder le problème. Une attention particulière a été portée à l'expérience anglaise, en raison de la procédure utilisée pour mettre au point les solutions (conférence de citoyens), et par ailleurs de la subtilité des solutions. Enfin, et de manière plus fondamentale, on examine la question de savoir si toute sélection des risques de santé, dans le cadre de l'assurance privée, peut constituer une discrimination au sens donné à cette notion complexe par les différentes branches du droit qui I'utilisent. Faute de pouvoir résumer de manière convaincante la totalité de l'étude, trois points méritent d'être développés : la légitimité du principe de bonne foi au regard du risque dit d'antisélection, I'expérience anglaise, la question de la discrimination.

\section{Bonne foi et antisélection}

Le principe de bonne foi, issu de plusieurs siècles de pratique de I'assu- rance, implique qu'il $n^{\prime} y$ ait pas $d^{\prime}$ 'asymétrie $d^{\prime}$ information entre les cocontractants. Cela concerne I'assureur qui doit décrire exactement les contours de son engagement, tout ce qui est assuré et tout ce qui est exclu. Le législateur et les tribunaux sont les gardiens de cette exigence. A l'inverse, l'assuré doit dire tout ce qui permet à l'assureur de forger son opinion du risque. Il le fait selon des procédures précises puisqu'il n'est tenu de répondre qu'aux questions qui lui sont posées et que l'assureur ne peut poser n'importe quelle question. Il y a donc une co-construction de l'objet du contrat et par voie de conséquence de son prix par le dialogue entre l'assureur et l'assuré à l'intérieur de contraintes légales : on n'assurera pas pour le même prix un homme jeune, fumeur, pratiquant des sports extrêmes et un jeune père de famille pratiquant la marche à pied; pour trouver le juste prix, il faut donc se renseigner sur le souscripteur.

\section{Le droit à ne pas subir un test génétique pour pouvoir s'assurer}

Un système totalement libéral fondé sur la seule idéologie du juste prix conduirait à reconnaître aux assureurs la liberté de concevoir des contrats très divers, assurant chacun au plus juste de son risque prévisible, après l'avoir soumis à des examens médicaux performants et une batterie de tests, génétiques ou non. L'investigation n'aurait pas de limite, les assureurs pouvant demander aux souscripteurs de réaliser toute une batterie de tests qu'ils n'auraient jamais songé à subir de leur propre chef. L'assurance ne doit évidemment pas jouer un rôle d'incitation économique qui pourrait perturber le consentement libre et éclairé de celui qui se soumet à un test. II faut donc bien affirmer I'interdiction de lier la souscription $d^{\prime}$ un contrat d'assurance à la réalisation d'un test génétique.

\section{L'obligation de fournir à I'assureur les résultats des tests connus de l'assuré}

Lorsqu'un individu a subi un test génétique, pour des raisons qui lui sont propres, et qu'il désire contracter une assurance, doit-il révéler le résultat ou non? Une réponse immédiate est fournie par le droit positif de l'assurance. La révélation n'est obligatoire que si le test est pertinent au regard du calcul du risque couvert. Or de nombreux tests qui pourraient intéresser les scientifiques n'intéresseront pas I'assureur.

Il faut déjà qu'ils soient totalement fiables sur le plan technique, et pertinents au regard du calcul du risque (c'est ainsi qu'au bout de quelques années d'observation des gènes $B R C A-1$ et BRCA-2, impliqués dans certains cancers du sein, la pertinence de la liaison entre la présence du gène muté et le déclenchement de la maladie est moins assurée qu'on ne le pensait à l'origine). Autrement dit, le résultat du test doit distinguer suffisamment l'individu du reste de la population pour que cela ait une incidence économique. Une augmentation de quelques points du risque d'être atteint d'un cancer par rapport à la population générale n'a pas de sens économique. Le calcul économique est luimême difficile à faire car un test sur un cancer rare, qui n'est pas pertinent pour l'assurance-vie qui concerne de larges populations et intègre de multiples paramètres, peut l'être pour un "produit» plus précis comme le risque «maladies redoutées», qui porte précisément sur le cancer ; c'est produit par produit qu'il faut apprécier la pertinence. Les choses peuvent encore être compliquées si l'on prend l'exemple des contrats d'assurance-vie à durée très courte, couvrant l'emprunt destiné à un achat de bien de consommation : s'agissant $d^{\prime}$ un contrat à deux ans, la présence du gène de chorée du Huntington n'a pas beaucoup de sens chez un individu de 25 ans, puisque la maladie se déclare entre 40 et 50 ans en moyenne.

On réalise d'emblée qu'il y a une distance considérable entre l'établissement d'une carte génétique à des fins assurantielles et I'utilisation rationnelle des données génétiques dans le cadre du droit positif. C'est donc dans ce cadre qu'il faut poser la question ; lorsque l'assuré dispose d'un résultat fiable et pertinent pour procéder au calcul du risque couvert, doit-il au nom du principe de bonne foi divulguer ce résultat à l'assureur ou faut-il envisager de mettre en place des législations lui interdisant de le faire ? L'argument des assureurs pour maintenir le droit positif est le risque d'antisélection. 


\section{L'antisélection}

Dans la branche «vie», l'assureur sélectionne les risques liés à la maladie, au handicap, à la mort, à la durée de la vie. Il le fait en anticipant comme il le peut le risque du souscripteur par rapport au risque moyen : tables d'espérance de vie, questionnaire sur la santé et les modes de vie, sports dangereux, etc. $\mathrm{Si}$ le questionnaire sur les pathologies antérieures, les interventions chirurgicales, I'histoire familiale, le ratio taille-poids, révèle des indices inquiétants, une enquête plus approfondie est menée à l'aide d'attestations, examen clinique, radios, analyses biologiques. L'assureur prend alors sa décision en fonction de "l'opinion» qu'il se fait de la santé du souscripteur : un risque "aggravé » conduit à une surprime (3\% des cas) ou à un refus d'assurance ( $1 \%$ des cas).

La précision croissante des outils de diagnostic a sur ce schéma des conséquences paradoxales. D'un côté, elle permet de révéler un avenir médiocre à des gens qui sont en parfaite santé : I'assureur y gagne, l'assuré y perd. Les tests génétiques renforceront et généraliseront de telles anticipations. Mais, en renseignant sur les risques de récidive d'un cancer, la faculté de réponse à une chimiothérapie, les risques de complication des différents types de diabète, ils permettent à l'inverse de repérer au sein de populations que I'on n'assurait pas du tout, des sujets assurables. Ici, des personnes qui n'étaient pas assurables le deviennent, et I'amélioration des connaissances scientifiques permet de réduire la proportion des exclus de l'assurance et $d^{\prime}$ assurer certains malades acceptant une surprime. Apparemment, le test génétique s'insère logiquement dans ce schéma sans le transformer : les outils de diagnostic seront plus nombreux, et surtout de plus en plus prédictifs au sens où ils renseigneront moins sur l'état de santé actuel que sur les perspectives de santé.

Certains tests offrent une certitude. Le souscripteur sait qu'il va être malade, même si la date de déclenchement et la gravité de l'atteinte restent incertaines. Les assureurs anglais ont déjà fait valoir leur intérêt pour certains de ces tests. Pourtant, quand on y réfléchit, on réalise qu'ils ne concernent que quelques maladies monogéniques et n'ont qu'une faible importance quantitative. Seules feront les tests les familles qui se savent à risque pour telle ou telle maladie. Le risque couru par l'assureur auquel on cache le résultat du test est très important si on le calcule opération par opération, mais modéré si on fait un calcul économique global, sauf pour certains produits spécifiques : si l'on prend l'exemple de l'assurance de la dépendance des personnes âgées qui n'a que peu de souscripteurs, la connaissance de la perspective d'une chorée de Huntington ou de diverses maladies neuro-dégénératives liées à l'âge peut concerner une part importante des souscripteurs, donc risquer d'atteindre l'équilibre économique du produit. $\mathrm{Ne}$ s'assureront que ceux qui se savent atteints. Le droit au mensonge paraît inopportun pour ce genre de produits, alors qu'il n'a probablement pas beaucoup d'impact pour l'assurance-vie.

Mais la plupart des tests, dans l'avenir, concerneront des maladies polygéniques et multifactorielles, pour lesquelles la présence du gène $n^{\prime}$ indique qu'une augmentation du risque par rapport à la population générale, et I'influence de l'environnement et des comportements peut être considérable. Du coup, I'interprétation de la présence du gène est difficile, et le jury de citoyens anglais a recommandé un moratoire sur ces tests. Les populations concernées sont quantitativement importantes, et ces gènes indicateurs de cancers et de maladies cardiovasculaires constituent la véritable cible de la médecine prédictive. Lorsque ces tests seront disponibles, les pouvoirs publics seront logiquement conduits à mener des campagnes de dépistage pour inciter les sujets à risque à adopter I'hygiène de vie qui leur convient. Dans un tel contexte de dépistages systématiques, une partie importante du public connaîtrait son profil génétique. Si les souscripteurs étaient protégés par un droit au mensonge, ils pourraient déduire de cette connaissance non partagée par l'assureur des stratégies de souscription qui seraient évidemment réservées aux profils médiocres ou mauvais. Le problème change alors de dimension, et le risque "d'antisélection» relevé par les assureurs, mérite d'être examiné. L'antisélection, ou sélection adverse, se produit lorsque les règles définissant les conditions de souscription d'un type déterminé de contrat conduisent à attirer une proportion anormale de "mauvais risques", alors que le prix est calculé pour un risque moyen. Les sinistres se multiplient, il faut augmenter les primes, ce qui conduit au désengagement croissant des «bons risques». Deux mécanismes y concourent, I'asymétrie d'information et l'aléa moral.

Les asymétries d'information disent bien leur nom : l'assuré conserve par-devers lui une information utile pour que de l'assureur forge son opinion du risque, et donc le prix. L'aléa moral s'observe chaque fois que le comportement de l'assuré intervient dans la survenance du risque ou l'ampleur du dommage. Ces phénomènes sont bien documentés : I'assurance «perte d'emploi », dont les conditions d'entrée avaient été mal délimitées, n'a guère attiré que des salariés se sachant menacés à court terme un milliard de pertes pour deux milliards de cotisations. Même phénomène avec un contrat destiné aux diabétiques : regroupant invalidité, décès, garantie emprunteur, il avait attiré les diabétiques ne sachant pas ou ne voulant pas équilibrer leurs modes de vie et leurs traitements, les autres s'en étant désintéressés. Cela se traduisit par une augmentation des primes de $100 \%$ et la renégociation du produit sur de nouvelles bases.

Dans le cas des tests génétiques, l'asymétrie d'information serait évidente si la loi accordait à l'assuré un droit au mensonge sur les résultats des tests qu'il a subis. Les assurances en cas de décès et les assurances concernant certains types de maladies seront souscrites par ceux qui connaissent I'aggravation de leur risque. Cela aura peut-être moins d'importance pour les assurances complémentaires de la sécurité sociale, car les causes de sinistres peuvent être si diverses que les tests génétiques perdent de leur importance. Ce seront donc surtout des produits très ciblés qui risquent d'être mis en péril. De même, l'aléa moral est évident pour les maladies multifactorielles dans lesquelles le comportement est essentiel : les personnes qui souhaitent manger n'importe quoi, fumer et boire, s'exposer au soleil, malgré des prédispositions défavorables pourraient être tentées plus que d'autres de s'assurer. $C^{\prime}$ est donc produit par produit qu'il faudrait prendre la décision. 


\section{La sélection des risques n'est pas une discrimination!}

Le Parlement français, stigmatisant les risques de discrimination, a choisi de punir d'un an d'emprisonnement et $100000 \mathrm{~F}$ d'amende "le fait de détourner de leurs finalités médicales ou de recherche scientifique les informations recueillies sur une personne au moyen de l'étude de ses caractéristiques génétiques »: liberté pour la science, censure des autres usages. La même idée d'un usage purement scientifique ou médical fut reprise par la convention européenne sur la bioéthique. Cependant, ces textes ne disent rien de I'enjeu véritable : car les assureurs sont $d^{\prime}$ accord pour ne pas détourner subrepticement des résultats, ni exiger la réalisation d'un test avant la souscription d'un contrat, mais une grande partie d'entre eux souhaitent appliquer le droit commun, qui oblige le souscripteur à divulguer tout ce qu'il sait si on lui en fait la demande. L'enjeu juridique de la révision des lois sur le bioéthique est bien là : faut-il instituer un droit au mensonge? Si l'on prenait ce parti, il y aurait asymétrie d'information entre les deux parties au contrat - I'un connaît le risque, l'autre pas - ; or, le contrat d'assurance, contrat de "bonne foi", implique l'information réciproque des deux partenaires, condition pour fixer le prix. Entre risque de discrimination pour raison de santé, et risque de déséquilibre économique des assurances de personnes, le débat n'est pas si simple à mener !

Malgré tout, lorsqu'en 1990, le Parlement vota une loi sur la prévention des discriminations contre les personnes du fait de leur état de santé, il fut décidé que la sélection des risques de santé par les assureurs ne constituait pas une telle discrimination mais l'exercice normal de leur métier, et cette disposition ne fut pas imposée à la sauvette mais effectivement discutée.

\section{L'évolution du droit des assurances: l'exemple anglais}

Il est difficile de se faire une idée de la manière dont va évoluer le droit français des assurances. L'utilisation des tests génétiques, outils de sélec- tion des risques, dépend finalement du modèle d'assurance de référence, plutôt libéral ou plutôt solidariste. Car il existe deux modèles d'assurance privée dont les valeurs s'affrontent. La France adhère traditionnellement au modèle solidariste qui implique de larges groupes d'assurés et mutualise des classes de risques diversifiées à la faveur d'assurances obligatoires et du développement des assurances de groupes: les bons risques payent pour les mauvais, ce qui est dans la nature de l'assurance, mais les bonnes classes de risques payent pour les mauvaises, ce qui n'est pas inéluctable. L'assuré ne joue pas sur la concurrence, ni entre les produits, ni entre les assureurs. Peu stimulé, celui-ci n'a pas à inventer des produits reposant sur une sélection fine des classes de risques.

La Grande-Bretagne et les Pays-Bas ont adopté le modèle libéral. Ici, le client change souvent d'assureur et de produit pour trouver le meilleur rapport qualité-prix correspondant à son profil de risque. Pour démultiplier leur offre, les assureurs segmentent les risques, fragmentent les populations pour que chacun y trouve sa niche. II y a bien un effet de solidarité puisque les bons risques payent pour les mauvais à l'intérieur d'une même classe de risque, mais l'objectif poursuivi est celui du juste prix : I'assuré veut payer selon sa classe de risque. C'est dans ce modèle que l'utilisation de tests génétiques s'intègre le plus nécessairement. Le droit français se rapprochant lentement du modèle anglais, le code de conduite élaboré par l'association des assureurs anglais ( $\mathrm{ABI}$ ) au moment où se déroulait un vaste débat et une conférence de citoyens n'est pas sans intérêt. II en ressort que :

- nul ne peut se voir contraint à effectuer un test, mais si un test a été effectué, le souscripteur doit en indiquer le résultat (sauf dans le cas des assurancesvie souscrites avec un prêt immobilier inférieur à un certain montant) ;

- I'assureur ne s'intéresse qu'aux tests dont la fiabilité et I'utilité pour le produit d'assurance concerné sont démontrées. C'est le Genetic advisor de I'ABI qui décide de cet intérêt (actuellement 8 tests sur les 70 qui sont sur le marché) : le test de la maladie d'Alzheimer est pertinent, pas celui de I'X fragile. En cas de conflit avec un souscripteur, celui-ci peut faire appel de la décision devant une instance indépendante, spécialement chargée de cette mission ; - un test positif peut entraîner une surprime, mais un test négatif n'entraînera pas de rabais, de manière à éviter la course aux rabais pour «bon profil génétique» - ce serait le levier le plus efficace de l'antisélection.

Il est difficile de savoir si les Français se reconnaîtraient dans cette approche. A la faveur du coup d'arrêt imposé à la croissance du système de sécurité sociale, un consensus politique s'est établi pour laisser l'assurance privée occuper le terrain, tout en lui imposant une certaine prise en compte de l'intérêt général : contrôle des exclusions, prise en charge des états pathologiques antérieurs à la souscription, etc. Cela n'empêche pas les assureurs français de s'essayer, eux aussi, à la fragmentation des marchés : c'est ainsi que I'on voit arriver des contrats pour les non-fumeurs pratiquant un sport doux, avec une économie de $80 \%$ sur le tarif habituel !

On peut donc se demander ce qu'il adviendra lors de la remise en chantier des lois sur la bioéthique. La crainte des scientifiques est que I'utilisation des résultats des tests génétiques par les assureurs conduise le public à ne pas réaliser ces tests. Cette perspective, qui légitimerait le droit au mensonge, n'est pas évoquée par les radios ou les analyses biologiques. II est vrai qu'elles sont réalisées uniquement lorsqu'une maladie est suspectée, et pas de manière préventive pour avoir une idée de l'avenir. Ce sont donc bien les campagnes de dépistage génétique qui créent un problème spécifique, dont il conviendrait d'examiner les conséquences au cas par cas

m/S2000
Summary
Insurers's use genetic tests
The use of genetic testing, pushing
predictive medicine to its utmost
limits, has aroused great concern
from the very outset. When genetic
testing is in the hands of insurers is
it just another tool or a more dange-
rous mechanism ? What will hap-
pen when laws on bioethics are
applied ? Scientists' fears centre on
popular rejection of these tests. Evi-
dence shows that screening campai-
gns are at the heart of the debate.

